\title{
Consideraciones sobre la desigualdad de oportunidades: nueva evidencia
}

\author{
Wallace Patrick Santos de Farias Souza, Ana Cláudia \\ Annegues y Victor Rodrigues de Oliveira
}

\section{Resumen}

El objetivo de este trabajo es evaluar los efectos de un conjunto de variables en la desigualdad de oportunidades en el Brasil. Para ello se utilizó el método desarrollado por Li, Chen y Gao (2011). Se combinaron datos de la Encuesta Nacional de Hogares (PNAD) y Finanzas de Brasil (Finbra) sobre los estados brasileños relativos al período comprendido entre 1995 y 2012. Los resultados indican que en los últimos años, el crecimiento económico ha perdido terreno en ese debate con respecto a los demás condicionantes. El patrón actual de los gastos en educación contribuye al mantenimiento de una situación de vulnerabilidad social que impide la plena integración de los individuos en la sociedad. Por otra parte, el aumento de la educación formal y de la formalización permitió una reducción de la desigualdad de oportunidades.

Palabras clave

Macroeconomía, crecimiento económico, igualdad de oportunidades, medición, modelos econométricos, Brasil

Clasificación JEL

D63, C14, C23

Autores

Wallace Patrick Santos de Farias Souza es Doctorando en Economía Aplicada del Programa de Posgrado en Economía de la Universidad Federal de Rio Grande do Sul (UFRGS), Brasil. wpsfarias@gmail.com

Ana Cláudia Annegues es Doctoranda en Economía Aplicada del Programa de Posgrado en Economía de la Universidad Federal de Rio Grande do Sul, Brasil. annegues.ana@gmail.com

Victor Rodrigues de Oliveira es Doctorando en Economía Aplicada del Programa de Posgrado en Economía de la Universidad Federal de Rio Grande do Sul, Brasil. victor5491@gmail.com 


\section{Introducción}

La libertad de elegir el número de horas de trabajo implica automáticamente que

la diferencia de ingresos deriva de elecciones de las que somos personalmente responsables. La responsabilidad es una consecuencia necesaria de cierto grado sustancial de libertad y, por lo tanto, forma parte de toda sociedad libre.

Fleurbaey (2008, pág. 1)

La desigualdad puede tener muchas dimensiones. En general, los economistas se preocupan específicamente por su dimensión monetaria, midiendo la desigualdad de ingresos individual o familiar. Sin embargo, la desigualdad puede entenderse de forma más amplia como el resultado del acceso desigual a las oportunidades de trabajo, la educación, la felicidad, la salud, a una mayor esperanza de vida, a activos y a la movilidad social, entre otros. La teoría de la desigualdad moderna, en la que se destacan los textos de Rawls (1971) y Roemer (1998), incorpora esas dimensiones en sus análisis¹.

Según este enfoque, más que la distribución final de los recursos, es importante tener en cuenta el proceso intermedio de asignación de esos recursos, que dependerá de los factores que determinan las ganancias económicas individuales. Esos factores son: el esfuerzo individual, medido por variables que los agentes pueden controlar, y las circunstancias, que están fuera del control de los individuos (raza o color, origen socioeconómico, entre otros). De acuerdo con el concepto de igualdad de oportunidades, la desigualdad de ingresos total estaría compuesta por la desigualdad originada por diferenciales de esfuerzo y por aquella derivada de factores de circunstancia fuera del control de los agentes. Solo esta última se consideraría de hecho injusta y debería ser, por lo tanto, el objetivo de las políticas públicas².

La igualdad de oportunidades y su medición no solo son relevantes desde el punto de vista normativo. En primer lugar, una cantidad cada vez mayor de evidencias empíricas muestra que las preferencias en materia de redistribución y la orientación política están moldeadas por preocupaciones de equidad. Por ejemplo, Alesina y Angeletos (2005) mostraron que, en el caso de los Estados Unidos, las personas que creen que el éxito económico individual se relaciona más con el esfuerzo que con la suerte tienen preferencias menores por la redistribución. Por otra parte, sobre la base de datos de la Encuesta Mundial sobre Valores, Alesina y La Ferrara (2005) encontraron que las percepciones de justicia están relacionadas con la orientación política de los individuos: cuando las personas creen que el esfuerzo es el principal determinante de las ventajas económicas, la redistribución y los impuestos son bajos, mientras que en las sociedades donde las personas creen que las condiciones iniciales (el nacimiento y las conexiones establecidas a partir de ese momento) son los principales determinantes del éxito económico, los impuestos y la redistribución serán mayores. En segundo lugar, visto que

\footnotetext{
1 En la literatura teórica se indica que la noción de igualdad de oportunidades incorpora dos principios básicos: el principio de compensación, que exige la eliminación de las desigualdades debidas a las circunstancias, y el principio de recompensa, que se refiere a la forma de recompensar los esfuerzos entre los individuos con circunstancias idénticas. El principio de compensación admite un punto de vista ex post o ex ante. El primero analiza el rendimiento real del individuo y se refiere a las diferencias de ingresos entre individuos con las mismas características de responsabilidad y circunstancias diferentes. El enfoque ex ante, en cambio, se concentra en las perspectivas, para que haya desigualdad de oportunidades si los individuos enfrentan diferentes conjuntos de oportunidades (o conjuntos de valores diferentes) a causa de sus circunstancias. Con respecto al principio de recompensa, en la literatura se distinguen la recompensa liberal y la recompensa utilitaria. En el primer caso se sostiene que el gobierno no debe redistribuir ingresos entre aquellos que comparten las mismas características de circunstancia, pues sus diferencias de ingresos se deben exclusivamente a las diferencias en los esfuerzos. En el segundo, se sostiene que no se debe preocupar por lo que solo se debe a las diferencias de esfuerzos. Véase un análisis detallado de esos puntos en Ramos y Van De Gaer (2012).

2 Schokkaert y Devooght (2003), Gaertner y Schwettmann (2007) y Cappelen, Sorenson y Tungodden (2010) proporcionaron sólidas pruebas de que, al juzgar la distribución del ingreso, las personas distinguen considerablemente las circunstancias y los esfuerzos, como sugieren las teorías de igualdad de oportunidades. Por ejemplo, Cappelen, Sorenson y Tungodden (2010) propusieron un ejercicio en el que se evalúan los elementos por los que las personas se sienten responsables. Los autores observaron que la gran mayoría de los participantes no atribuía a las personas la responsabilidad por el precio determinado aleatoriamente, un factor impersonal que está más allá del control del individuo, pero las responsabilizaban por su elección del tiempo de trabajo.
} 
los determinantes de la desigualdad económica (circunstancias y esfuerzos) influyen en los incentivos individuales, esos determinantes están relacionados con los resultados económicos agregados, como el crecimiento económico. En el Informe sobre el Desarrollo Mundial 2006, el Banco Mundial sostiene que la desigualdad de ingresos debida a las circunstancias puede derivar en una acumulación subóptima de capital humano y, por lo tanto, a un menor crecimiento económico, mientras que la desigualdad de ingresos debida a variables relacionadas con la responsabilidad individual puede incentivar a las personas a invertir en capital humano y a realizar el mayor esfuerzo posible (Banco Mundial, 2005).

En las últimas décadas se registró una reducción constante de los índices de desigualdad del Brasil, no obstante todavía esté en la lista de los países más desiguales del mundo (Barros y otros, 2007) ${ }^{3}$. En la esfera social, se adoptaron programas de combate a la pobreza y la miseria de forma moderada en 1995, pero con gran énfasis a partir de $2003^{4}$. Las políticas públicas de acceso universal a la educación ${ }^{5}$ y a la salud básica también desempeñan un papel destacado en la reducción de las disparidades brasileñas ${ }^{6}$. Además del crecimiento y de la mayor focalización de los programas sociales, las reformas macroeconómicas que tuvieron lugar en el país a partir de la primera mitad de la década de 1990, como la estabilización monetaria y la apertura comercial, también pueden haber contribuido a la reducción de la desigualdad de ingresos brasileña. A partir del Plan Real se observa una creciente valorización del salario mínimo, que incidió directamente en el nivel de bienestar de las familias, sobre todo las más pobres. Aunque de forma limitada, la combinación de esos factores permite comprender que el Brasil presente altas tasas de concentración del ingreso y grandes disparidades regionales, además de que, en períodos históricos diferentes, el crecimiento económico tuviera efectos distintos en la reducción de la desigualdad de ingresos, la pobreza y la desigualdad de oportunidades.

A pesar de que el ambiente macroeconómico desempeña un papel importante desde el punto de vista social, en los estudios sobre el Brasil se ha prestado poca atención a ese tema para concentrarse más en el impacto de los programas sociales como el Bolsa Família ${ }^{7}$. En la literatura internacional, ese debate tiene lugar desde hace algún tiempo, con un número cada vez mayor de estudios en los que se procura investigar los efectos de los factores macroeconómicos en los indicadores sociales. Las variables macroeconómicas más estudiadas se relacionan con el crecimiento económico y la inflación.

El debate sobre la relación entre desigualdad y crecimiento económico comienza con la formulación teórica de la curva de Kuznets (1955), que postulaba una relación no lineal entre ambas variables, descrita por una curva en forma de "U" invertida. La idea es que la distribución de los ingresos sufriría un empeoramiento en las etapas iniciales del desarrollo mientras que más tarde los incrementos de productividad se extenderían internamente y, de ese modo, la desigualdad tendería a disminuir. Desde entonces, no se ha llegado a una conclusión en la literatura sobre la verdadera naturaleza de esa relación. Alesina y Rodrik (1994) realizan una regresión de la tasa de crecimiento media anual con la desigualdad inicial, medida por el coeficiente de Gini, sobre la base de datos de corte transversal relativos a distintos países. Los resultados muestran que la desigualdad de ingresos

3 En la lista de factores que contribuyen a las variaciones de la desigualdad de ingresos se destacan particularmente la educación, la raza, los programas sociales, la región y la demografía espacial (Neri, 2011).

4 Véase un análisis detallado de los programas actuales de combate a la pobreza y las futuras estrategias en Rocha (2007).

5 Las principales transformaciones de la enseñanza pública brasileña incluyen la descentralización de los recursos educativos y la expansión de las matrículas a partir de 1996, que culminaron en la creación del Fondo de Mantenimiento y Desarrollo de la Enseñanza Fundamental y Valorización del Magisterio (FUNDEF).

6 Menezes-Filho, Fernandes y Picchetti (2007) señalaron que a partir de 1997, con el rápido aumento de la proporción de jóvenes en la enseñanza media, el efecto composición y el efecto compresión (diferencial de salarios) comenzaron a reducir la desigualdad de ingresos.

7 Avila, Bagolin y Comim (2012, pág. 461) cuestionan la validez de los programas de transferencias, mostrando que, por lo menos en los segmentos con cierto nivel de ingresos (bajo), los incrementos monetarios no se traducen necesariamente en una mejora de las condiciones multidimensionales de los individuos, es decir que los ingresos monetarios por sí solos son insuficientes para caracterizar las privaciones humanas. 
está inversamente relacionada con el crecimiento subsiguiente. Por otra parte, Li y Zou (1998) y Forbes (2000) utilizaron paneles con efectos fijos y concluyeron que la desigualdad de ingresos tendría una relación positiva con el crecimiento económico. En otros enfoques se habla de los efectos positivos del crecimiento mediante el acceso de los agentes al mercado de trabajo (Nolan, 1987) y, más recientemente, Ravallion (2012) mostró que los niveles iniciales de pobreza estarían relacionados con bajas tasas de crecimiento económico.

Con respecto a la inflación, se argumenta que su efecto adverso en la desigualdad de ingresos se debe al aumento de precios, que afecta considerablemente a los más pobres. En algunos trabajos teóricos se trata de sistematizar este argumento mediante el desarrollo de modelos de equilibrio general, como los trabajos de Erosa y Ventura (2002) y de Cysne, Maldonado y Monteiro (2005). Algunos resultados no son concluyentes y se relacionan con el origen del proceso inflacionario: si el aumento de precios obedece a presiones en la oferta, la desigualdad disminuiría, mientras que, si la inflación deriva de presiones por el lado de la demanda, esta empeoraría (Blinder y Esaki, 1978; Buse, 1982).

En este estudio se incorpora un conjunto de variables a la lista de determinantes de la desigualdad generada por las circunstancias, algunas de las cuales se basan en el análisis empírico realizado por Marrero y Rodríguez (2010). Si bien los programas sociales concentran la mayor atención cuando se habla de políticas de reducción de la desigualdad, el ambiente económico desempeña un papel importante (incluso viabilizando o no las medidas de política social), de manera que conviene medir empíricamente el impacto de las variables que lo conforman.

La medición de la desigualdad injusta se basa en la estimación de un modelo en el que los ingresos dependen de variables de circunstancia y esfuerzo. Se utilizan el logaritmo de los salarios reales (variable sustitutiva del ingreso individual) como variable dependiente y un conjunto de variables explicativas que representan características específicas de los individuos, incluida la decisión de migrar. Dado que el proceso migratorio es autoselectivo, se utiliza el método propuesto por Nelsen (2006) ${ }^{8}$ para evitar estimaciones sesgadas de los salarios. A continuación se calculan los componentes de la desigualdad total sobre la base de los ingresos ajustados del modelo, manteniendo constantes las variables de circunstancia (desigualdad de esfuerzo) y esfuerzo (desigualdad de circunstancia). En esta etapa se calculan los índices de desigualdad de oportunidades mediante el coeficiente de Gini.

A partir de los índices calculados se construye un panel de datos para el período de 1996 a 2012 con información por estado. Después se utiliza un conjunto de variables para verificar el impacto en la desigualdad de circunstancias construida como se describió anteriormente, que se considera la única parte de la desigualdad socialmente indeseable.

Las variables utilizadas siguen los enfoques ya presentes en la literatura: el producto interno bruto (PIB) real per cápita como indicador de crecimiento, los gastos per cápita en salud y educación, la media de años de estudio de hombres y mujeres y el grado de informalidad de la economía. Para ello se utiliza el método propuesto por Li, Chen y Gao (2011) para estimar el impacto de esas variables en el nivel de desigualdad. Este método permite lidiar con ese problema, pues se trata de un enfoque no paramétrico, en el cual no se impone una forma funcional específica que describa el comportamiento de los datos. Además, es apropiado para el empleo de datos en panel, pues elimina los efectos fijos sin necesidad de las variables en primera diferencia.

El trabajo se divide en cuatro secciones además de esta Introducción. En la segunda se presentan los procedimientos empíricos adoptados y en la tercera se describen las bases de datos. En la cuarta sección se justifican las variables utilizadas y se presentan los resultados de las estimaciones. Por último, en la quinta sección se realizan las consideraciones finales del estudio.

8 Véanse aplicaciones a los casos internacional y nacional, respectivamente, en Meng (2001) y Ramalho y Queiroz (2011). 


\section{Estrategia empírica}

Para evaluar la manera en que las variables citadas anteriormente se relacionan con la desigualdad de oportunidades se utiliza el método propuesto por Li, Chen y Gao (2011). Si bien la literatura sobre panel de datos es amplia, las especificaciones paramétricas pueden conducir a modelos subespecificados $y$, en consecuencia, a estimadores incongruentes. Para superar ese problema se realizaron diversos estudios sobre ese aspecto (Ullah y Roy, 1998; Fan y Li, 2004; Henderson, Carroll y Li, 2008; Zhang, Fan y Sun, 2009). Al mismo tiempo, en los últimos años surgió una línea de investigación con el objetivo de modelar series temporales no estacionarias. Gao y Hawthorne (2006) mostraron que los modelos en que la tendencia lineal se obtiene mediante una especificación paramétrica no presentan un buen ajuste. Una de las principales características de los modelos no paramétricos es que los datos "hablan por sí mismos". En ese sentido, Gao y Hawthorne (2006) y Atak, Linton y Xiao (2011) utilizaron esa estrategia para determinar la forma funcional de la tendencia en un contexto de modelos de panel de datos y series temporales. Sin embargo, se ha prestado poca atención a las series temporales no paramétricas con coeficientes variables en el tiempo ${ }^{9}$ y mucho menos para modelos de panel. Uno de los primeros trabajos en incorporar estos aspectos fue propuesto por Robinson (2012). No obstante, Li, Chen y Gao (2011) desarrollaron un método que permite estimar la tendencia no lineal y los coeficientes de las variables explicativas sin utilizar la primera diferencia para eliminar los efectos fijos.

Para comprender el método utilizado en este artículo se supone que la variable dependiente, $Y_{i t}$, se modela de la siguiente manera:

$$
\begin{gathered}
Y_{i t}=f_{t}+\sum_{j=1}^{d} \beta_{t, j} X_{i t, j}+\alpha_{i}+\varepsilon_{i t} \\
Y_{i t}=f_{t}+\sum_{j=1}^{d} X_{i t}^{T} \beta_{t}+\alpha_{i}+\varepsilon_{i t}, i=1, \ldots, N, t=1, \ldots, T
\end{gathered}
$$

en que $X_{i t}=\left(X_{1 t, 1}, \ldots, X_{1 y, d}\right)^{T}, \beta_{t}=\left(\beta_{t, 1}, \ldots, \beta_{t, d}\right)^{T}, f_{t}$ y $\beta_{t}$ son funciones desconocidas, $\left\{\alpha_{i}\right\}$ es el efecto individual no observado y $\left\{\varepsilon_{i}\right\}$ es un proceso débilmente dependiente y estacionario para cada observación $i$ e independiente de $\left\{X_{i t}\right\}$ y $\left\{\alpha_{i}\right\}$ con $E\left[\varepsilon_{i t}\right]=0$ y $E\left[\varepsilon_{i t}^{2}\right]=\sigma_{\varepsilon}^{2} 10$. Se supone que $\left\{\alpha_{i}\right\}$ está correlacionado con $\left\{X_{i t}\right\}$, es decir, un modelo con efectos fijos. Se asume que el efecto fijo satisface la siguiente condición ${ }^{11}$ :

$$
\sum_{i=1}^{N} \alpha_{i}=0
$$

Se supone que la función $f_{t}$ y el coeficiente de vectores $\beta_{t}$ satisfacen las siguientes condiciones:

$$
f_{t}=f\left(\frac{t}{T}\right) \text { y } \beta_{t, j}=\beta_{j}\left(\frac{t}{T}\right) t=1, \ldots, T
$$

\footnotetext{
9 En muchas áreas de investigación en las que se necesita un amplio conjunto de estadísticas existen algunos modelos que se utilizan tradicionalmente. Sin embargo, a menudo estos ignoran la dinámica subyacente al conjunto de datos, pese a que el estudio de dicha característica a veces puede ser muy atractivo. Para examinar esa característica dinámica y mejorar el ajuste del modelo, se permite que los parámetros evolucionen en el tiempo. Estos modelos fueron introducidos por Cleveland, Grosse y Shyu (1991).

${ }^{10}$ El modelo impone homocedasticidad, pero es posible incorporar heterocedasticidad. Véanse detalles en Li, Chen y Gao (2011).

11 Esta condición es idéntica a la asumida por Sun, Carroll y Li (2009).
} 
en que $f(\cdot)$ y $\beta_{j}(\cdot)$ son funciones continuas desconocidas. Así, se analizarán dos estimadores, que eliminan el efecto fijo de distinta manera.

\section{Estimación lineal local (averaged local linear estimation)}

Para introducir este estimador se define:

$$
Y_{. t}=\frac{1}{N} \sum_{i=1}^{N} Y_{i t}, X_{. t}=\frac{1}{N} \sum_{i=1}^{N} X_{i t} \text { y } \varepsilon_{. t}=\frac{1}{N} \sum_{i=1}^{N} \varepsilon_{i t}
$$

Al tomar la media en $i$ y usar $\sum_{i=1}^{N} \alpha_{i}=0$, se obtiene:

$$
Y_{. t}=f_{t}+X_{. t}^{T} \beta_{t}+\varepsilon_{. t}, \quad t=1, \ldots, T
$$

en la cual se eliminan los efectos individuales $\alpha_{i}$ 's. Al establecer las siguientes notaciones: $Y_{. t}=\left(Y_{.1}, \ldots, Y_{. T}\right)^{T}, \quad f=\left(f_{1}, \ldots, f_{T}\right)^{T}, \quad B(X, \beta)=\left(X_{.1}^{T} \beta_{1}, \ldots, X_{. T}^{T} \beta_{T}\right)^{T}$ y $\quad \varepsilon=\left(\varepsilon_{.1}, \ldots, \varepsilon_{. T}\right)^{T}$, el modelo (4) puede reescribirse de la siguiente manera:

$$
Y=f+B(X, \beta)+\varepsilon
$$

Se utiliza la formulación del estimador lineal local propuesta por Fan y Gijbels (1996) para estimar $\beta_{*}()=.\left(f(.), \beta_{1}(.), \ldots, \beta_{d}(.)\right)^{T}$.

Para un $0<\tau<1$ dado, se define

$$
M(\tau)=\left(\begin{array}{ccccc}
1 & X_{.1}^{T} & \frac{1-\tau T}{T h} & \frac{1-\tau T}{T h} & X_{.1}^{T} \\
\vdots & \vdots & \vdots & \vdots & \vdots \\
1 & X_{. T}^{T} & \frac{T-\tau T}{T h} & \frac{T-\tau T}{T h} & X_{. T}^{T}
\end{array}\right)
$$

y

$$
W(\tau)=\operatorname{diag}\left[K\left(\frac{1-\tau T}{T h}\right), \ldots, K\left(\frac{T-\tau T}{T h}\right)\right]
$$

en que K es la función Kernel y h es el ancho de banda.

Asumiendo que $\beta *(\cdot)$ tiene derivadas continuas hasta el segundo orden, mediante la expansión de Taylor se obtiene:

$$
\beta_{*}\left(\frac{t}{T}\right)=\beta_{*}(\tau)+\beta_{*}^{\prime}(\tau)\left(\frac{t}{T}-\tau\right)+O\left[\left(\frac{t}{T}-\tau\right)^{2}\right]
$$

en que $0<\tau<1$ y $\beta_{*}^{\prime}($. $)$ es la derivada de $\beta_{*}(\cdot)$. A partir de la aproximación establecida por (8), el estimador lineal local de $\beta_{*}^{T}($. ) es 


$$
\hat{\beta}_{*}(\tau)=\left[I_{d+1}, O_{d+1}\right]\left[M^{T}(\tau) W(\tau) M(\tau)\right]^{-1} M^{-1}(\tau) W(\tau) Y
$$

en que $I_{d+1}$ es una matriz identidad $(d+1) \times(d+1)$ y $0_{d+1}$ es una matriz nula $(d+1) \times(d+1)$. El ancho de banda se selecciona por validación cruzada.

\section{Enfoque de variable ficticia lineal local (local linear dummy variable approach)}

$\mathrm{Li}$, Chen y Gao (2011) establecieron un estimador alternativo que presenta una tasa de convergencia más rápida. Para ello, se reescribe el modelo (1) de la siguiente manera:

$$
\tilde{Y}=\tilde{f}+\tilde{B}(X, \beta)+\tilde{D} \alpha+\tilde{\varepsilon}
$$

en que

$$
\begin{gathered}
\tilde{Y}=\left(Y_{1}^{T}, \ldots, Y_{N}^{T}\right)^{T}, Y_{i}=\left(Y_{i 1}, \ldots, Y_{i T}\right)^{T}, \\
\tilde{f}=\bar{I}_{N} \otimes\left(f_{1}, \ldots, f_{T}\right)^{T}=\bar{I}_{N} \otimes, \\
\tilde{B}(X, \beta)=\left(X_{11}^{T} \beta_{1}, \ldots, X_{1 T}^{T} \beta_{T}, X_{21}^{T} \beta_{1}, \ldots, X_{N T}^{T} \beta_{T}\right)^{T} \\
\tilde{D}=I_{N} \otimes \bar{I}_{T}, \\
\alpha=\left(\alpha_{1}, \ldots, \alpha_{N}\right)^{T}, \\
\tilde{\varepsilon}=\left(\varepsilon_{1}^{T}, \ldots, \varepsilon_{N}^{T}\right)^{T}, \varepsilon_{i}=\left(\varepsilon_{i 1}, \ldots, \varepsilon_{i T}\right)^{T},
\end{gathered}
$$

$\otimes$ es el operador de Kronecker, $I_{k}$ es un vector de dimensión $k$ de 1 y $f$ se define como en (4).

Al usar la condición de identificación, la ecuación (10) puede reescribirse de la siguiente manera:

$$
\tilde{Y}=\tilde{f}+\tilde{B}(X, \beta)+\tilde{D}^{*} \alpha^{*}+\tilde{\varepsilon}
$$

en que $\alpha^{*}=\left(\alpha_{2}, \ldots, \alpha_{N}\right)^{T}$ y $\tilde{D}^{*}=\left(-\bar{I}_{N-1}, I_{N-1}\right)^{T} \otimes \bar{I}_{T}$. A partir de la expansión de Taylor establecida en la ecuación (8), se obtiene:

$$
\tilde{f}+\tilde{B}(X, \beta) \approx \tilde{M}(\tau)\left\{B_{*}^{T}(\tau), h\left[\beta_{*}^{\prime}(\tau)\right]^{T}\right\}^{T}
$$

en que $\beta_{*}(\cdot)=\left[f(\cdot), \beta_{1}(\cdot), \ldots, \beta_{d}(\cdot)\right]^{T}$ y $\bar{M}^{T}(\tau)=\left[M_{1}^{T}(\tau), \ldots, M_{N}^{T}(\tau)\right]$ con

$$
M_{I}(\tau)=\left(\begin{array}{ccccc}
1 & X_{i 1}^{T} & \frac{1-\tau T}{T h} & \frac{1-\tau T}{T h} & X_{i 1}^{T} \\
\vdots & \vdots & \vdots & \vdots & \vdots \\
1 & X_{i T}^{T} & \frac{T-\tau T}{T h} & \frac{T-\tau T}{T h} & X_{i T}^{T}
\end{array}\right)
$$


El estimador de $\beta *(\tau)$ es

$$
\tilde{\beta}_{*}(\tau)=\left[I_{d+1} O_{d+1}\right]\left[\tilde{M}^{T}(\tau) \tilde{W}^{*}(\tau)\right]^{-1} \tilde{M}^{-1}(\tau) \tilde{W}^{*} \tilde{Y}
$$

en que $\tilde{W}^{*}(\tau)=I_{N} \otimes W(\tau)$.

El ancho de banda se selecciona por validación cruzada modificada conforme la propuesta de Sun, Carroll y Li (2009), a diferencia de su forma estándar para el modelo propuesto en la sección anterior, es decir,

$$
\hat{h}^{o p t}=\arg _{h} \min \left[Y-B\left(X, \tilde{\beta}_{(-1)}\right)\right]^{T} M_{D}^{T} M_{D}\left[Y-B\left(X, \tilde{\beta}_{(-1)}\right)\right]
$$

en que $M_{D}=I_{N \times T}-\frac{1}{T} I_{N} \otimes\left(\varepsilon_{T} \varepsilon_{T}^{T}\right)$ satisface $M_{D} I_{N} \otimes \varepsilon_{T}=0^{12}$.

\section{Datos}

Para lograr el objetivo propuesto se utilizaron dos fuentes de datos, a saber: la Encuesta Nacional de Hogares (PNAD) y Finanzas de Brasil (Finbra) de la Secretaría del Tesoro Nacional.

La PNAD es realizada por el Instituto Brasileño de Geografía y Estadística (IBGE) en todo el territorio nacional, por muestreo probabilístico de hogares. La encuesta, que se realiza desde fines de la década de 1960, cuenta con un cuestionario de carácter permanente que incluye preguntas relativas a características del hogar y las personas, como el tamaño de la familia, los ingresos del hogar y el nivel educativo de los habitantes, entre otras. En algunos años se investigan características socioeconómicas y demográficas complementarias, como la migración, la salud, la seguridad alimentaria y otros temas. Cabe destacar que el estudio promovió el ajuste de la muestra con el objetivo de respetar el plan de muestreo que le dio origen ${ }^{13}$.

Se utilizaron los datos relativos al período 1995-2012 ${ }^{14}$. Los salarios del trabajo se deflactaron mediante el índice nacional de precios al consumidor (INPC) para septiembre de $2012^{15}$. Se consideró a los individuos de entre 25 y 65 años de edad y a los que eran jefes de familia con el objetivo de mitigar la heterogeneidad de la muestra utilizada.

En primer lugar, se estima el salario de los trabajadores como una función de un conjunto de variables, a saber: sexo, color, experiencia en el mercado de trabajo, años de estudio, migración, situación familiar (casado y sin hijos, casado con hijos menores de 14 años y madre con hijos menores de 14 años), condición en el mercado de trabajo (sin contrato de trabajo formal, autónomo, empleador, funcionario público y empleado por cuenta propia) y variables ficticias de carácter geográfico (región urbana o rural, región metropolitana y estados $\left.{ }^{16}\right)$.

\footnotetext{
12 Esta condición elimina el efecto fijo que es desconocido.

13 El plan (diseño) de muestreo de la PNAD incorpora todos los aspectos que definen un "plan de muestreo complejo": estratificación de las unidades de muestreo, conglomeración (selección de la muestra en varias etapas, con unidades compuestas de muestreo), probabilidades desiguales de selección en una o más etapas y ajustes de los pesos muestrales para calibración con totales de población conocidos. Por ese motivo, los datos obtenidos mediante las muestras de las PNAD generalmente no pueden tratarse como si fueran observaciones independientes e idénticamente distribuidas (es decir, como si hubieran sido generados por muestras aleatorias simples con reposición). Véanse detalles en Nascimento Silva, Pessoa y Lila (2002).

${ }^{14}$ Los datos de 2000 y 2010 no fueron empleados en este estudio, pues en esos años no se realizó la encuesta de la PNAD junto con los censos.

15 Véanse detalles de la deflación en Corseuil y Foguel (2002).

${ }^{16}$ La muestra utilizada no incluye el Distrito Federal, pues no se disponía de los datos de Finbra relativos a algunos años.
} 
Sin embargo, el modelo teórico propuesto por Borjas y Bratsberg (1996) establece que el proceso de migración es autoselectivo, pues esa decisión depende del rendimiento relativo del salario, condicionado al conjunto de habilidades. Así, se utiliza una estrategia de parametrización conjunta de los determinantes de la migración y de los salarios. Se asume que el individuo puede elegir entre migrar y no migrar. Por medio de un modelo estructural de utilidad aleatoria, se obtiene que el beneficio neto de elegir la alternativa $m$ está representado por:

$$
U_{m}=\delta_{m} \ln G_{m}+k_{m} Z_{m}+v_{m}
$$

en que $\ln G_{m}$ es el salario esperado para la opción $m$ (expresado en logaritmo natural), $Z m$ es un conjunto de características, $k_{m}$ y $\delta_{m}$ son vectores que han de estimarse y $v_{m}$ es un término de error aleatorio.

Además, para cada opción disponible para el individuo $i$, existe una ecuación minceriana como la siguiente:

$$
\ln G_{m}=\mu_{m} R_{m}+u_{m}
$$

Al sustituir (17) en (16), se obtiene:

$$
U_{m}^{*}=\mu_{m} \delta_{m} X_{m}+k_{m} Z_{m}+\delta_{m} u_{m}+v_{m}
$$

en que $U_{m}^{*}$ es una variable latente que mide el beneficio neto de la opción $m$. Para corregir el sesgo de selección, Nelsen (2006) propuso estimar (18) por medio del uso de cópulas ${ }^{17}$. Para ello, sea la siguiente ecuación de selección:

$$
S_{i}=\left\{\begin{array}{l}
0, \text { si } S_{i}^{*}=z_{i} \gamma^{\prime}+\varepsilon_{s i} \leq 0, \\
1, \text { si } S_{i}^{*}=z_{i} \gamma^{\prime}+\varepsilon_{s i}>0 .
\end{array}\right.
$$

en que $S_{i}$ es un indicador de selección y $z_{i}$ es un vector de covariables.

La variable de resultado sigue la estructura siguiente:

$$
y_{i}=x_{i}^{\prime} \beta+\varepsilon_{1 i}
$$

Como $\varepsilon_{i s}$ y $\varepsilon_{1 i}$ no son independientes, una regresión por mínimos cuadrados ordinarios (MCO) resultaría en estimaciones sesgadas de $\beta$. A partir de esta estructura, la función de log-verosimilitud es:

$$
L=\prod_{i=1}^{N}\left\{\int_{-\infty}^{-z_{i}^{\prime} \gamma} f_{s}\left(\varepsilon_{s}\right) d \varepsilon s\right\}^{S_{i}=0}\left\{\int_{-z_{i}^{\prime} \gamma}^{\infty} f_{s 1}\left(\varepsilon_{s}, \varepsilon_{1 i}\right) d \varepsilon s\right\}^{S_{i}=1}
$$

en que $f_{s j}$ es la función de densidad de probabilidad de $\varepsilon_{s}$ y $\varepsilon_{j}$ para $j=0,1$. De la ecuación (20), se deduce que:

\footnotetext{
${ }^{17}$ En ciencias estadísticas, particularmente en la teoría de la probabilidad, el concepto de cópula se define como una función de distribución multivariada cuyas densidades marginales se distribuyen uniformemente.
} 


$$
\begin{gathered}
\int_{-\infty}^{-z_{i}^{\prime} \gamma} f_{s}\left(\varepsilon_{s}\right) d \varepsilon s=F_{s}\left(-z_{i}^{\prime} \gamma\right) \\
\int_{-z_{i}^{\prime} \gamma}^{\infty} f_{s 1}\left(\varepsilon_{s}, \varepsilon_{1 i}\right) d \varepsilon s=\left.\frac{\partial}{\partial \varepsilon_{1}}\left\{F_{1}\left(\varepsilon_{1}\right)-F_{s 1}\left(-z_{i}^{\prime}, \varepsilon_{1}\right)\right\}\right|_{\varepsilon_{1}=\varepsilon_{1 i}}
\end{gathered}
$$

Para implementar esta estructura por medio de cópulas, sean $\omega_{1}$ y $\omega_{2}$ dos variables aleatorias. Se asume que $u_{i}=F_{i}\left(\omega_{i}\right)$ es la función de densidad marginal de $\omega_{i}$ para $i=1,2$ y que $F\left(\omega_{1}, \omega_{2}\right)$ es la función de distribución conjunta. La cópula $C(\cdot)$ permite la obtención de $F\left(\omega_{1}, \omega_{2}\right)$ por medio de las densidades marginales, es decir,

$$
F\left(\omega_{1}, \omega_{2}\right)=C\left\{F_{1}\left(\omega_{1}\right), F_{2}\left(\omega_{2}\right) ; \theta\right\}=C\left\{u_{1}, u_{2} ; \theta\right\}
$$

en que $\theta$ mide el grado de dependencia.

Para implementar esta estrategia, se necesita la derivada parcial de la función de distribución conjunta, es decir,

$$
\frac{\partial}{\partial \omega_{1}} F\left(\omega_{1}, \omega_{2}\right)=\frac{\partial}{\partial u_{1}} C\left\{u_{1}, u_{2} ; \theta\right\} \times \frac{\partial F_{1}\left(\omega_{1}\right)}{\partial \omega_{1}}
$$

Así, la función de verosimilitud, ecuación (20), se reescribe de la siguiente manera:

$$
L=\prod_{i=1}^{N}\left[F_{s}\left(-z_{i}^{\prime} \gamma\right)\right]^{S_{i}=0}\left[\left\{1-\frac{\partial}{\partial u_{1}} C\left\{u_{1 i}, u_{s i} ; \theta_{1}\right\}\right\} \times f_{1}\left(\varepsilon_{1 i}\right)\right]^{S_{i}=1}
$$

Como hay muchas cópulas que pueden utilizarse, la ecuación (22) se estimará para diferentes cópulas y el modelo final se elegirá sobre la base de criterios de información.

A partir de los salarios ajustados por esas variables se calcula el coeficiente de Gini de la desigualdad injusta. Una vez obtenidas las medidas de desigualdad por estado y por año, se emplea el método descrito en la sección anterior. En esta etapa, en que se procura evaluar los determinantes de la desigualdad de oportunidades, se utilizaron las siguientes variables explicativas, conforme el trabajo de Marrero y Rodríguez (2010) ${ }^{18}$ : los logaritmos del PIB per cápita, del gasto per cápita en educación, del gasto per cápita en salud, la media de estudio de hombres y mujeres (en años) y la tasa de informalidad de la economía (porcentaje de individuos sin contrato de trabajo formal) ${ }^{19}$.

En el cuadro A1.2 del anexo se presenta la medida de desigualdad de oportunidades de todos los estados en el período 1995-2012. Se observa que en los estados pertenecientes a la región Norte esta desigualdad se mantuvo, con excepción del estado de Tocantins (donde se registró una disminución del 11,82\%). En la región Nordeste se observa el fenómeno opuesto, con la reducción sustancial de los índices en los estados de Pernambuco, Piauí y Ceará. También se observa una reducción de los índices en todos los estados de las regiones Sudeste, Sur y Centro-Oeste. En el gráfico 1 se muestra la evolución de la desigualdad en las regiones brasileñas entre 1995 y 2012. Si bien en todas las regiones hubo una tendencia descendente, se registró un incremento del índice de la región Nordeste al final del período de análisis.

\footnotetext{
18 Los valores correspondientes al PIB y a los gastos en educación y salud se deflactaron mediante el índice nacional de precios al consumidor amplio (IPCA), utilizándose la media anual.

19 Véanse las estadísticas descriptivas de las variables utilizadas en el cuadro A1.3 del anexo.
} 


\section{Gráfico 1}

Brasil: evolución de la desigualdad de oportunidades, 1995-2012

(En porcentajes)

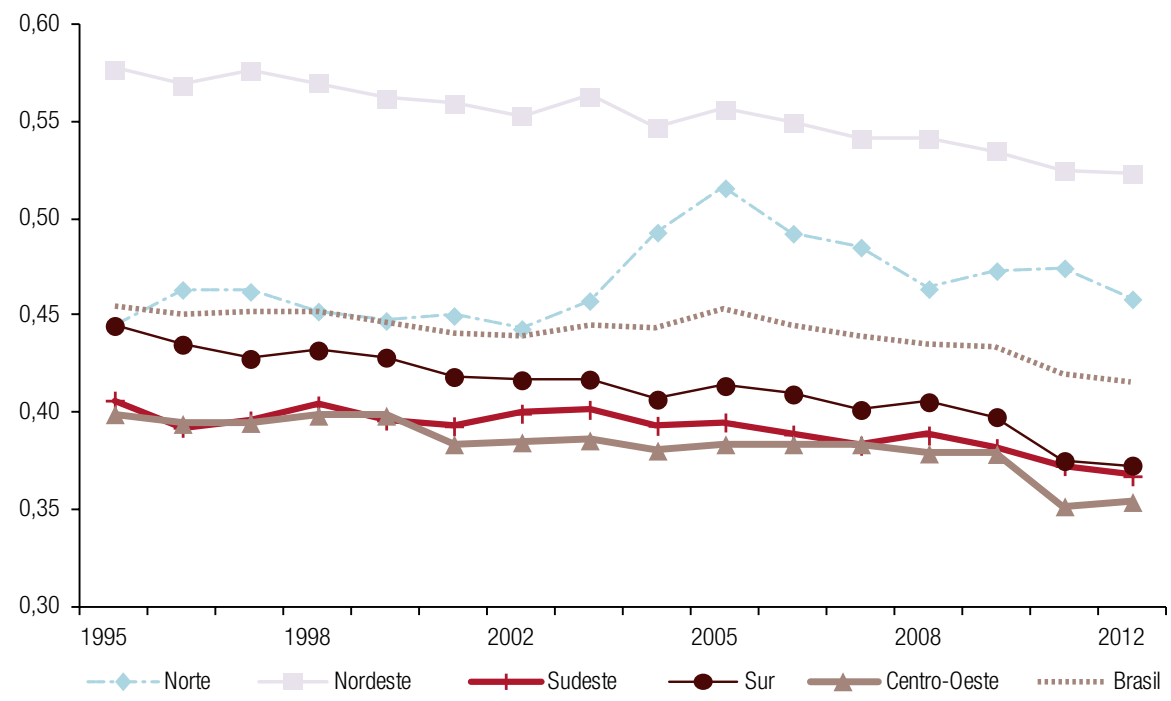

Fuente: Elaboración propia.

Los resultados obtenidos en el análisis de la evolución de la desigualdad de oportunidades son similares a los encontrados por Silva y otros (2013) con respecto a los municipios brasileños. Como indican los autores, los estados pertenecientes a las regiones Norte y Nordeste, conocidos por sus situaciones de gran vulnerabilidad social, presentaron un grado de desigualdad de oportunidades superior a la media brasileña. A partir de la metodología propuesta en este trabajo, y de acuerdo con la lista de variables de Marrero y Rodríguez (2010), se observaron altos índices de desigualdad injusta en el Nordeste en todo el período analizado, un resultado que corrobora la evidencia presentada por Silva y otros (2013). Por otra parte, se encontró que el nivel de la desigualdad que no puede atribuirse a las decisiones individuales es considerablemente superior a las estimaciones obtenidas en el estudio de Silva y otros (2013).

En la siguiente sección se presentan los resultados para los determinantes de la desigualdad de oportunidades 20 .

\section{Resultados}

En una primera etapa se corrigió la autoselectividad del proceso de migración. Para ello, las cópulas seleccionadas mediante el criterio de información de Akaike, a partir de la ecuación (22), fueron las siguientes: FGM para el año 1997, Plackett para el año 2003, AMH para el año 2007, Gaussiana para 2002 y 2005 y Frank para los demás años (véase el cuadro A1.1 del anexo). Después de esa etapa se calculó el efecto de las variables seleccionadas en la desigualdad de oportunidades por medio del estimador propuesto por Li, Chen y Gao (2011). La siguiente ecuación presenta el modelo utilizado para estimar el impacto de cada una de las variables descritas en la desigualdad de oportunidades.

\footnotetext{
20 Como los resultados de los dos estimadores propuestos por Li, Chen y Gao (2011) fueron muy similares, se optó por presentar solo los resultados obtenidos mediante la variable ficticia lineal local, conforme la sección II.2. Los resultados del otro estimador pueden solicitarse a los autores.
} 


$$
\begin{aligned}
Y_{i t}= & f\left(\frac{t}{T}\right)+\beta_{1}\left(\frac{t}{T}\right) X_{i t, 1}+\beta_{2}\left(\frac{t}{T}\right) X_{i t, 2}+\beta_{3}\left(\frac{t}{T}\right) X_{i t, 3}+\beta_{4}\left(\frac{t}{T}\right) X_{i t, 4} \\
& +\beta_{5}\left(\frac{t}{T}\right) X_{i t, 5}+\beta_{6}\left(\frac{t}{T}\right) X_{i t, 6}+\alpha_{i}+\varepsilon_{i t}, 1 \leq i \leq N, 1 \leq t \leq T
\end{aligned}
$$

en que $Y_{i t}$ es la medida de desigualdad de oportunidades, $X_{i t, 1}$, es el logaritmo del PIB real per cápita, $X_{i t, 2}$ es el logaritmo del gasto real en salud per cápita, $X_{i t, 3}$ es el logaritmo del gasto real en educación per cápita, $X_{i t, 4}$ es la media de años de estudio de los hombres, $X_{i t, 5}$ es la media de años de estudio de las mujeres y $X_{i t, 6}$ es el grado de informalidad de la economía.

EI PIB real per cápita es una de las variables comúnmente utilizadas en la literatura para investigar el papel de los factores macroeconómicos en la determinación de la desigualdad, pues afecta las condiciones económicas de los individuos. El crecimiento de la economía se traduce en la inclusión de un número mayor de trabajadores en el mercado laboral, que a su vez deriva en la reducción de la desigualdad de resultados (Metcalf, 1969; Mirer, 1973; Powers, 1995). También en la lista de las variables macroeconómicas, los gastos per cápita en salud y educación afectan los ingresos de los individuos, sobre todo los menos favorecidos, porque contribuyen al aumento de su productividad. La evidencia empírica de Marrero y Rodríguez (2010) mostró que el aumento de esos gastos tuvo un impacto negativo en los índices de desigualdad.

La media de años de estudio de hombres y mujeres da una idea de la productividad de cada individuo. Al igual que las inversiones per cápita en educación, el crecimiento de la media de estudios de la población suele tener un efecto positivo en la productividad media de los trabajadores. En consecuencia, aumentan los ingresos y su distribución es más equitativa.

El modelo teórico de Chong y Gradstein (2007) sugiere que hay una relación positiva entre la desigualdad de ingresos y el grado de informalidad de la economía. En un ambiente con instituciones de baja calidad y en el que no se respetan los derechos de propiedad, los individuos menos favorecidos no logran extraer una gran parte de los recursos producidos, de manera que acaban por migrar al sector informal, donde pueden retener la totalidad de los frutos de su producción. El mercado informal presenta una baja productividad con respecto al sector formal, lo que fomentaría un mayor grado de desigualdad de ingresos en la economía.

En el gráfico 2 se presentan los efectos de las variables mencionadas por medio del estimador de la variable ficticia lineal local. El gráfico está compuesto por seis gráficos en los que se muestra el impacto de cada una de las variables utilizadas en la desigualdad de oportunidades. En el gráfico 2A se muestra la correlación entre el PIB per cápita y el índice de desigualdad. Si bien el gráfico parece indicar una relación positiva, el coeficiente estimado es negativo. Esto indica que al inicio del período de análisis las condiciones de ingresos tenían una importancia significativa en la reducción de la desigualdad. Sin embargo, después de ese período y con el dinamismo de la economía brasileña a partir de 2003, otros factores comenzaron a cobrar importancia relativa en la explicación de la evolución de la desigualdad de oportunidades y redujeron la primacía del crecimiento económico. Mediante matrices de transición de Markov, Magalhães y Miranda (2009) mostraron que el ingreso per cápita presenta un grave proceso de divergencia en el Brasil, caracterizado por el desplazamiento de la mayoría de los municipios a solo dos grupos: el club de los ricos - formado mayoritariamente por los municipios del Sur, el Centro-Oeste y el Sudeste, con un ingreso per cápita entre 1,27 y 1,68 veces superior a la media de todos los municipios - y el club de los pobres - constituido por los municipios del Norte y el Nordeste, con un ingreso per cápita de hasta 0,55 de dicha media-. Boueri y otros (2007), por su parte, mostraron que la distribución ergódica en el período siguiente comienza a sugerir una tendencia a la convergencia. Por último, en la década más reciente, los resultados de largo plazo indican una concentración de municipios y de población en las clases intermedias de la distribución. 


\section{Gráfico 2}

Estimación de la desigualdad según distintas variables y coeficiente de Gini, 1995-2012

A. Correlación entre

el índice de desigualdad

y el PIB per cápita

$$
\left(\beta_{1}\left(\frac{t}{T}\right) X_{i t, 1}\right)
$$

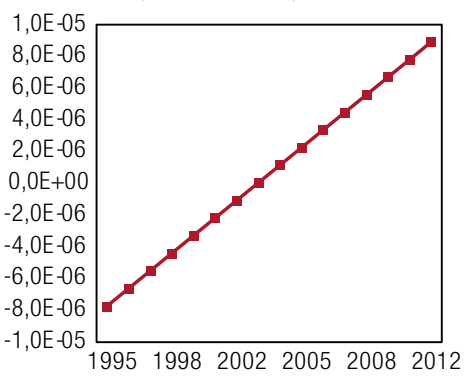

D. Correlación entre la desigualdad de oportunidades

y los años de educación de los hombres

$$
\left(\beta_{4}\left(\frac{t}{T}\right) X_{i t, 4}\right)
$$

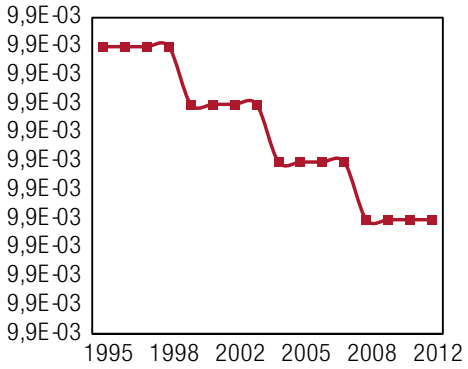

B. Correlación entre la desigualdad injusta $\mathrm{y}$ los gastos en salud

$$
\left(\beta_{2}\left(\frac{t}{T}\right) X_{i t, 2}\right)
$$

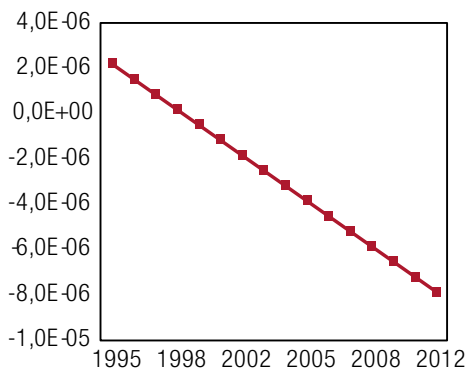

E.Correlación entre la desigualdad de oportunidades y los años de educación de las mujeres

$$
\left(\beta_{5}\left(\frac{t}{T}\right) X_{i t, 5}\right)
$$

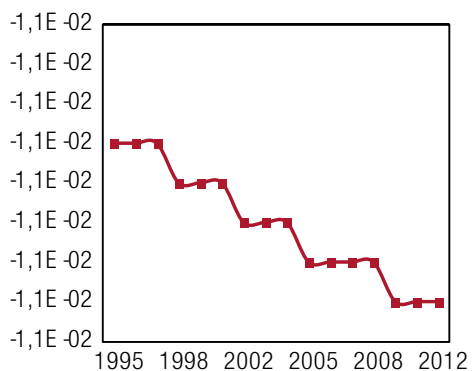

C. Correlación entre la desigualdad injusta y los gastos en educación

$$
\left(\beta_{3}\left(\frac{t}{T}\right) X_{i t, 3}\right)
$$

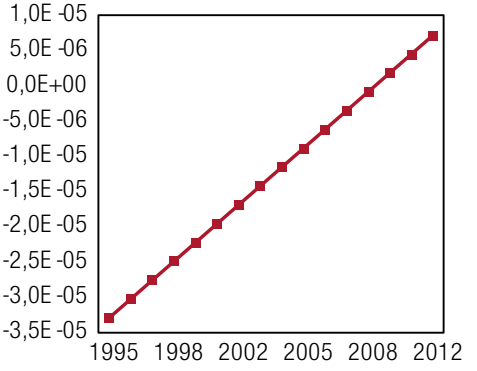

F. Correlación entre la desigualdad de oportunidades y la tasa de informalidad de la economía

$$
\left(\beta_{6}\left(\frac{t}{T}\right) X_{i t, 6}\right)
$$

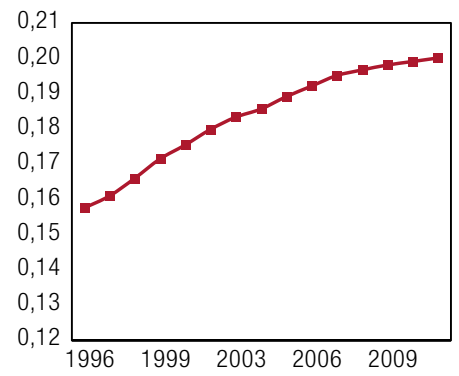

Fuente: Elaboración propia.

Nota: Los valores del eje vertical representan el impacto de una variable con una unidad de escala muy superior a la de la desigualdad de oportunidades. PIB: Producto interno bruto.

La relación entre el crecimiento económico y la reducción de la desigualdad de oportunidades es compleja e importante. Por ese motivo, es necesario entender los canales por los que el crecimiento económico actúa sobre los individuos. No se debe olvidar que los avances en el bienestar, en última instancia, dependen del crecimiento económico y de la manera en que los individuos aprovechan sus beneficios. Sin embargo, el carácter del crecimiento económico es importante, pues puede actuar en forma neutra, a favor o en contra de los individuos. Por consiguiente, las estrategias para promover la reducción de la desigualdad de oportunidades no pueden basarse exclusivamente en el crecimiento económico, sino que deben combinarse con políticas de redistribución del ingreso y con gastos públicos adecuados.

En los gráficos 2B y 2C se muestra la correlación entre la desigualdad injusta y los gastos en salud y en educación, respectivamente.

Las características del sector de salud y los cambios en la estructura etaria y el patrón de mortalidad y morbilidad del Brasil tienen consecuencias en los costos y la utilización de los servicios médicos y ambulatorios. Además, las transformaciones en los sistemas de salud -reorganización de los modelos de atención - y la incorporación de nuevas tecnologías, entre otros factores, también han modificado los patrones de utilización de esos servicios. Esas dinámicas modificaron el patrón 
de gasto en salud en los últimos años. Los cambios en este sector pueden analizarse mediante un enfoque microeconómico. Desde el punto de vista individual, el consumo de bienes y servicios de salud afecta directamente el bienestar en la medida en que el estado de salud determina el grado de felicidad de los individuos. De forma similar, desde un punto de vista macroeconómico, la provisión de servicios de salud incide en el crecimiento económico, pues compone el acervo de capital humano y determina la capacidad productiva de la economía. De esa forma, la relación entre ellos puede observarse tanto en el mercado de trabajo, visto que se trata de un sector intensivo en mano de obra para los servicios de baja y media complejidad, como en la producción, visto que es intensivo en tecnología para los sectores de alta complejidad. Así, la importancia de la inversión en salud, cualquiera sea el enfoque, puede verse en términos de bienestar social. Por lo tanto, los efectos de estos cambios se sienten en la asignación de los recursos y en la organización de la infraestructura de los servicios de salud, lo que demuestra la relevancia de la provisión de estos bienes y servicios y sus efectos en las oportunidades individuales. En el gráfico 2B se observa que la relación es negativa en todos los períodos analizados, de manera que las inversiones en salud tienen un papel importante en la igualación de las oportunidades entre los individuos.

En el gráfico 2C se presenta la relación entre los gastos per cápita en educación y la desigualdad de oportunidades, que muestra una asociación positiva. Este resultado deriva en gran parte de la falta de eficiencia en la utilización de políticas de responsabilidad y rendición de cuentas. Estas cobraron importancia en el debate sobre la gestión de los gastos públicos, aunque de forma incipiente, pero no lograron alcanzar las metas ni dar equidad al sistema educativo. En ese sentido, el fracaso de la educación brasileña, tanto en materia de inversiones directas como de calidad de la enseñanza ofrecida, contribuye a ese resultado. Estos factores contribuyen a mantener una situación de vulnerabilidad social que impide la plena integración de los individuos en la sociedad.

En los gráficos 2D y 2E se muestra la manera en que la evolución de los años de educación formal de hombres y mujeres, respectivamente, incide en las oportunidades de los individuos. Se observa una relación clara y directa entre esas variables. En el gráfico 3 se muestra que los estados en los que se registró la mayor reducción de la desigualdad de oportunidades fueron aquellos que presentaron, de forma general, las mayores tasas de crecimiento de la educación formal de hombres y mujeres. Ese resultado indica que el aumento de la escolaridad media permitió incrementos salariales (de forma directa) y una reducción de la tasa de depreciación del acervo de salud (de manera indirecta), que también contribuye positivamente al rendimiento en el mercado de trabajo. La suma de esos dos factores permite que los individuos tengan mayor acceso a oportunidades y, así, el efecto de las circunstancias se compensa mediante el esfuerzo individual. Es necesario destacar que la dimensión monetaria de la desigualdad de oportunidades, captada por el coeficiente de Gini, es un enfoque parcial y limitado. Aunque insuficiente, el enfoque de la desigualdad de oportunidades basada en los ingresos no debe descuidarse, pues la privación de ingresos representa una de las principales causas de la disminución del acceso a condiciones apropiadas de educación, salud y seguridad, entre otras.

En la década de 1990 hubo una reducción constante de los puestos de trabajo considerados formales (empleados con contrato de trabajo) en el Brasil. Sin embargo, a partir de la década siguiente se registró un incremento en la proporción de trabajadores con registro formal de trabajo. En el gráfico 2F se muestra que la tasa de informalidad de la economía (porcentaje de individuos sin contrato de trabajo formal) está directamente relacionada con la desigualdad de oportunidades, es decir, el aumento de la formalización de la economía permitió una reducción de esa desigualdad. Se observa una tendencia creciente de la importancia de esa variable y esa relación también se verifica en el gráfico 4. 


\section{Gráfico 3}

Brasil: tasa de crecimiento de los años de estudio y de la desigualdad de oportunidades, 1995-2012

(En porcentajes de variación)

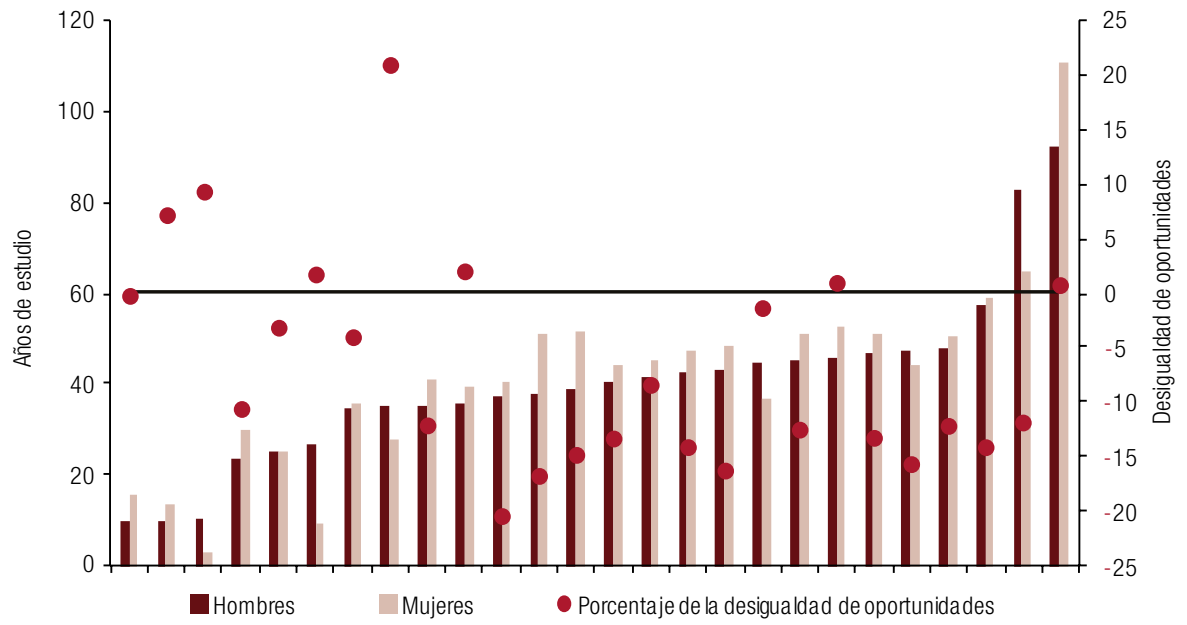

Fuente: Elaboración propia.

Gráfico 4

Brasil: evolución de la informalidad y de la desigualdad de oportunidades, por estado, 1995-2012

(En porcentajes de variación)

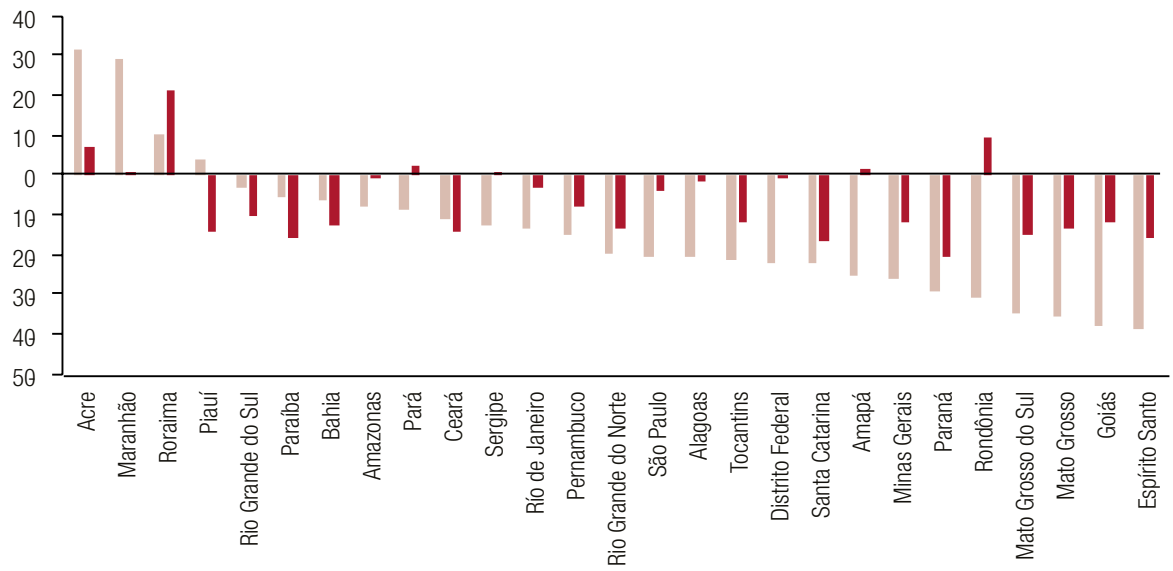

Fuente: Elaboración propia.

Sin contrato de trabajo formal _Desigualdad de oportunidades

\section{Conclusión}

El objetivo de este trabajo era evaluar los efectos de algunas variables macroeconómicas en la desigualdad de oportunidades. Para ello se adoptó una estrategia empírica en dos etapas. En primer lugar se corrigió la autoselectividad de la migración por medio de cópulas, conforme la propuesta de Nelsen (2006). En segundo lugar, después de corregir la ecuación de salarios, se estimó la relación entre un conjunto de variables macroeconómicas y la desigualdad de oportunidades resultante de los salarios ajustados. En esa etapa se utilizó el método desarrollado por Li, Chen y Gao (2011). Se combinaron datos de la PNAD y Finbra sobre los estados brasileños relativos al período comprendido entre 1995 y 2012. 
Los resultados indican que en los últimos años el crecimiento económico ha perdido terreno en ese debate con respecto a los demás condicionantes macroeconómicos. Este resultado puede obedecer a la dificultad para mantener un crecimiento sostenible y a la formación de grupos de estados que se distanciaron en los últimos años, pero que presentaron una inversión de esa tendencia. Al mismo tiempo, los gastos en salud están negativamente correlacionados con la desigualdad de oportunidades, si bien no se verifica esa relación en el caso de los gastos en educación. En ese sentido, las estrategias de reducción de las disparidades individuales deben combinar el aumento de los ingresos con gastos públicos de calidad. El patrón actual de los gastos en educación contribuye al mantenimiento de una situación de vulnerabilidad social que impide la plena integración de los individuos en la sociedad. Por otra parte, el aumento de la educación formal permitió una reducción de la desigualdad de oportunidades, por medio de un efecto directo en el salario y un efecto indirecto en el estado de salud. Por último, la creciente formalización de la economía brasileña, cuya intensidad aumentó a partir de la década de 2000, también contribuye considerablemente a la reducción de la desigualdad de oportunidades.

\section{Bibliografía}

Alesina, A. y G.M. Angeletos (2005), "Fairness and redistribution", American Economic Review, vol. 95, № 4, Nashville, Tennessee, American Economic Association.

Alesina, A. y E. La Ferrara (2005), "Preferences for redistribution in the land of opportunities", Journal of Public Economics, vol. 89, № 5-6, Amsterdam, Elsevier.

Alesina, A. y D. Rodrik (1994), "Distributive politics and economic growth", Quarterly Journal of Economics, vol. 109, No 2, Oxford University Press.

Atak, A., O. Linton y Z. Xiao (2011), "A semiparametric panel model for unbalanced data with application to climate change in the United Kingdom", Journal of Econometrics, vol. 164, № 1, Amsterdam, Elsevier.

Avila, R.P., I.P. Bagolin y F.V. Comim (2012), "Heterogeneidades individuais versus intensidade da pobreza em Porto Alegre - RS", Economia, vol. 13, № 2, Asociación Nacional de Centros de Posgrado en Economía (ANPEC).

Banco Mundial (2005), Informe sobre el desarrollo mundial 2006: equidad y desarrollo, Washington, D.C.

Barros, R.P. y otros (2007), "A queda recente da desigualdade de renda no Brasil", Desigualdade de renda no Brasil: uma análise da queda recente, R.P. Barros, M.N. Foguel y G. Ulyssea (eds.), vol. 1, Brasilia, Instituto de Investigación Económica Aplicada (IPEA).

Blinder, A.S. y H.Y. Esaki (1978), "Macroeconomic activity and income distribution in the postwar United States", Review of Economics and Statistics, vol. 60, № 4, Cambridge, Massachusetts, The MIT Press.

Borjas, G.J. y B. Bratsberg (1996), "Who leaves? The outmigration of the foreign-born", The Review of Economics and Statistics, vol. 87, № 1, Cambridge, Massachusetts, The MIT Press.

Boueri, R. y otros (2007), "Dinâmica espacial do crescimento econômico brasileiro (1970-2010)", Brasil em desenvolvimento 2013: estado, planejamento e políticas públicas, R. Boueri y M.A. Costa (eds.), Brasilia, Instituto de Investigación Económica Aplicada (IPEA).

Buse, A. (1982), "The cyclical behaviour of the size distribution of income in Canada 1947-1978", Canadian Journal of Economics, vol. 15, № 2, Canadian Economics Association.

Cappelen, A.W., E.O. Sorenson y B. Tungodden (2010), "Responsibility for what? Fairness and individual responsibility", European Economic Review, vol. 54, №3, Amsterdam, Elsevier.

Chong, A. y M. Gradstein (2007), "Inequality and informality", Journal of Public Economics, vol. 91, № 1, Amsterdam, Elsevier.

Cleveland, W.S., E. Grosse y W.M. Shyu (1991), "Local regression models", Statistical Models in S., J. Chambers y T.J. Hastie (eds.), Pacific Grove, Wadsworth \& Brooks.

Corseuil, C.H. y M.N. Foguel (2002), "Uma sugestão de deflatores para rendas obtidas a partir de algumas pesquisas domiciliares do IBGE”, Texto para Discussão, № 897, Río de Janeiro, Instituto de Investigación Económica Aplicada (IPEA).

Cysne, R.P., W. Maldonado y P.K. Monteiro (2005), "Inflation and income inequality: a shopping-time approach", Journal of Development Economics, vol. 78, № 2, Amsterdam, Elsevier. 
Erosa, A. y G. Ventura (2002), "On inflation as a regressive consumption tax", Journal of Monetary Economics, vol. 49, N 4, Amsterdam, Elsevier.

Fan, J. e I. Gijbels (1996), Local Polynomial Modelling and Its Applications, Londres, Chapman and Hall.

Fan, J. y R. Li (2004), "New estimation and model selection procedures for semiparametric modeling in longitudinal data analysis", Journal of the American Statistical Association, vol. 99, № 467, Taylor \& Francis.

Fleurbaey, M. (2008), Fairness, Responsibility, and Welfare, Nueva York, Oxford University Press.

Forbes, K. (2000), "A reassessment of the relationship between inequality and growth", American Economic Review, vol. 90, № 4, Nashville, Tennessee, American Economic Association.

Gaertner, W. y L. Schwettmann (2007), "Equity, responsibility and the cultural dimension", Economica, vol. 74, $N^{\circ} 296$, Wiley.

Gao, J. y K. Hawthorne (2006), "Semiparametric estimation and testing of the trend of temperature series", Econometrics Journal, vol. 9, $\mathrm{N}^{\circ}$ 2, Wiley.

Henderson, D., R.J. Carroll y Q. Li (2008), "Nonparametric estimation and testing of fixed effects panel data models", Journal of Econometrics, vol. 144, № 1, Amsterdam, Elsevier.

Kuznets, S. (1955), "Economic growth and income inequality", American Economic Review, vol. 45, № 1, Nashville, Tennessee, American Economic Association.

Li, D., J. Chen y J. Gao (2011), "Non-parametric time-varying coefficient panel data models with fixed effects", Econometrics Journal, vol. 14, No 3, Wiley.

Li, H. y H. Zou (1998), "Income inequality is not harmful for growth: theory and evidence", Review of Development Economics, vol. 2, №3, Wiley.

Magalhães, J.C. y R.B. Miranda (2009), "Dinâmica da renda per capita, longevidade e educação nos municípios brasileiros", Estudos econômicos, vol. 39, № 3, São Paulo.

Marrero, A.G. y J.G. Rodríguez (2010), "Inequality of opportunity and growth", Working Papers, No 154, ECINEQ.

Menezes-Filho, N., R. Fernandes y P. Picchetti (2007), "Educação e queda recente da desigualdade no Brasil”, Desigualdade de renda no Brasil: uma análise da queda recente, R.P. Barros, M.N. Foguel y G. Ulyssea (eds.), vol. 2, Brasilia, Instituto de Investigación Económica Aplicada (IPEA).

Meng, X. (2001), "The informal sector and rural-urban migration - a Chinese case study", Asian Economic Journal, vol. 15, № 1 , Wiley.

Metcalf, C.E. (1969), "The size distribution of personal income during the business cycle", American Economic Review, vol. 59, № 4, Nashville, Tennessee, American Economic Association.

Mirer, T.W. (1973), "The effects of macroeconomic fluctuation on the distribution of income", Review of Income and Wealth, vol. 19, N ${ }^{\circ} 4$, Wiley.

Nascimento Silva, P.L., D.G.C. Pessoa y M.F. Lila (2002), "Statistical analysis of data from PNAD: incorporating the sample design”, Ciência \& Saúde Coletiva, vol. 7, № 4, Río de Janeiro, Asociación Brasileña de Salud Colectiva.

Nelsen, R.B. (2006), An Introduction to Copulas, Nueva York, Springer.

Neri, M. (2011), Desigualdade de renda na década, Río de Janeiro, FGV Editora.

Nolan, B. (1987), "Cyclical fluctuations in factor shares and the size distribution of income", Review of Income and Wealth, vol. 33, N², Wiley.

Powers, E. (1995), "Inflation, unemployment, and poverty revisited", Economic Review, № 2, Cleveland, Banco de la Reserva Federal de Cleveland.

Ramalho, H.M.B. y V.S. Queiroz (2011), "Migração interestadual de retorno e autosseleção: evidências para o Brasil", Pesquisa e Planejamento Econômico, vol. 41, N3 3, Instituto de Investigación Económica Aplicada (IPEA).

Ramos, X. y D. Van De Gaer (2012), "Empirical approaches to inequality of opportunity: principles, measures, and evidence", IZA Discussion Paper, № 6672, Instituto para el Estudio del Trabajo (IZA).

Ravallion, M. (2012), "Why don't we see poverty convergence?", American Economic Review, vol. 102, № 1, Nashville, Tennessee, American Economic Association.

Rawls, J. (1971), A Theory of Justice, Cambridge, Harvard University Press.

Robinson, P.M. (2012), "Nonparametric trending regression with cross-sectional dependence", Journal of Econometrics, vol. 169, № 1, Amsterdam, Elsevier.

Rocha, S. (2007), "Os 'novos' programas de transferências de renda: impactos possíveis sobre a desigualdade no Brasil", Desigualdade de renda no Brasil: uma análise da queda recente, R.P. Barros, M.N. Foguel y G. Ulyssea (eds.), vol. 2, Brasilia, Instituto de Investigación Económica Aplicada (IPEA).

Roemer, J.E. (1998), Equality of Opportunity, Cambridge, Massachusetts, Harvard University Press. 
Schokkaert, E. y K. Devooght (2003), "Responsibility-sensitive fair compensation in different cultures", Social Choice and Welfare, vol. 21, $\mathrm{N}^{\circ} 2$, Springer.

Silva, V. y otros (2013), "Uma proposta para mensuração dos índices de desigualdade de oportunidade municipais" [en línea] http://www.ccsa.ufpb.br/ppge/arquivos/ensaios/td15_2013.pdf.

Sun, Y., R.J. Carroll y D. Li (2009), "Semiparametric estimation of fixed effects panel data varying coefficient models", Advances in Econometrics, vol. 25, Emerald.

Ullah, A. y N. Roy (1998), "Nonparametric and semiparametric econometrics of panel data", Handbook of Applied Economics and Statistics, A. Ullah y D.E.A. Giles (eds.), Nueva York, Marcel Dekker.

Zhang, W., J. Fan e Y. Sun (2009), "A semiparametric model for cluster data", Annals of Statistics, vol. 37, $N^{\circ} 2$, Institute of Mathematical Statistics.

\section{Anexo A1}

Cuadro A1.1

Resultados del empleo de cópulas para corregir la autoselectividad de la migración, 1995-2012

\begin{tabular}{|c|c|c|c|c|c|c|c|c|c|c|}
\hline \multirow{2}{*}{ Año } & \multicolumn{8}{|c|}{ Criterio de información de Akaike } & \multirow{2}{*}{$\begin{array}{c}\text { Cópula } \\
\text { seleccionada }^{a}\end{array}$} & \multirow{2}{*}{$\theta$} \\
\hline & Gaussiana & FGM & Plackett & $\mathrm{AMH}$ & Frank & Clayton & Gumbel & Joe & & \\
\hline 1995 & 48059,71 & 48056,75 & 48053,76 & 48047,60 & 48044,45 & 48059,28 & 48114,64 & 48187,19 & Frank & 0,27 \\
\hline 1996 & 47520,12 & 47502,70 & 47523,15 & 47521,48 & 47490,60 & 47513,63 & 47508,93 & 47647,91 & Frank & 0,33 \\
\hline 1997 & 51717,29 & 51680,52 & 51713,59 & 51681,52 & 51715,23 & 51714,77 & 51750,27 & 51897,91 & FGM & $-0,89$ \\
\hline 1998 & 50574,84 & 50573,07 & 50575,04 & 50573,10 & 50571,26 & 50574,59 & 50734,43 & 50739,76 & Frank & 0,08 \\
\hline 1999 & 51681,11 & 51672,20 & 51647,59 & 51639,06 & 51615,59 & 51676,01 & 51715,85 & 51893,31 & Frank & 0,41 \\
\hline 2001 & 58775,96 & 58773,69 & 58773,95 & 58773,64 & 58773,12 & 58773,99 & 58842,08 & 59007,08 & Frank & 0,02 \\
\hline 2002 & 62605,30 & 62606,21 & 62606,21 & 62606,20 & 62608,21 & 62608,21 & 62707,29 & 62818,08 & Gaussiana & 0,04 \\
\hline 2003 & 59862,64 & 59862,11 & 59854,56 & 59862,11 & 59861,94 & 59862,19 & 60001,84 & 60081,74 & Plackett & 0,17 \\
\hline 2004 & 62795,18 & 62772,10 & 62781,08 & 62789,63 & 62762,61 & 62798,06 & 62911,15 & 63044,55 & Frank & 0,35 \\
\hline 2005 & 30 236,94 & 30237,76 & 30237,74 & 30237,76 & 30237,37 & 30237,68 & 30345,47 & 30325,17 & Gaussiana & $-0,05$ \\
\hline 2006 & 69608,50 & 69579,25 & 69619,57 & 69575,62 & 69548,41 & 69 630,96 & 69791,28 & 69691,63 & Frank & 0,40 \\
\hline 2007 & 67346,47 & 67343,70 & 67346,40 & 67 338,88 & 67344,33 & 67343,86 & 67446,72 & 67602,64 & AMH & $-0,06$ \\
\hline 2008 & 71689,75 & 71685,56 & 71690,11 & 71688,38 & 71683,67 & 71690,03 & 71786,05 & 71870,61 & Frank & 0,08 \\
\hline 2009 & 71421,38 & 71416,94 & 71394,93 & 71 405,32 & 71392,06 & 71422,50 & 71558,94 & 71661,80 & Frank & 0,32 \\
\hline 2011 & 57894,80 & 57855,66 & 57895,93 & 57894,48 & 57852,15 & 57893,46 & 57962,15 & 58 066,96 & Frank & $-0,40$ \\
\hline 2012 & 59 163,44 & 59161,87 & 59 163,91 & 59 161,87 & 59 153,91 & 59 163,03 & 59313,24 & 59361,31 & Frank & $-0,31$ \\
\hline
\end{tabular}

Fuente: Elaboración propia.

a Véanse detalles sobre las funciones cópulas y el cálculo del $\theta$ de Kendall en Nelsen (2006). 
Cuadro A1.2

Brasil: coeficiente de Gini de la desigualdad injusta por estado, 1995-2012

\begin{tabular}{|c|c|c|c|c|c|c|c|c|c|c|c|c|c|c|c|c|}
\hline o & 5 & 96 & $I$ & 8 & 99 & 001 & 002 & 03 & 04 & 005 & 2006 & 2007 & 2008 & 009 & 011 & 012 \\
\hline Acre & & 3 & 3 & 5 & 5 & 8 & 5 & 0,432 & 6 & 3 & 5 & 7 & 2 & 17 & 3 & \\
\hline & & 2 & 199 & 73 & 0,456 & 0,511 & 0,479 & 08 & 58 & 30 & 549 & 488 & 07 & 25 & 64 & 501 \\
\hline 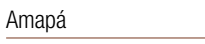 & 14 & 0,436 & 0,457 & 0,455 & 0,396 & 0,433 & 0,390 & 0,414 & 0,399 & 0,426 & 0,419 & 0,426 & 417 & 445 & 400 & 421 \\
\hline Amazonas & 79 & 0,363 & 0,386 & 0,368 & 0,361 & 0,392 & 0,390 & 0,391 & 0,413 & 0,426 & 0,433 & 0,391 & 0,382 & 0,380 & 417 & 378 \\
\hline Bahia & 69 & 0,578 & 0,529 & 0,548 & 0,546 & 0,528 & 0,539 & 0,547 & 20 & 537 & 0,541 & 0,518 & 0,529 &, 524 & 518 & 498 \\
\hline & 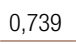 & 21 & 0,715 & 0,707 & 0,713 & 0,698 & 0,661 & 0,649 & 0,648 & 0,672 & 0,624 & 0,606 & 0,616 & 0,616 & 0,613 & 0,635 \\
\hline & 01 & 269 & 0,272 & 0,274 & 0,277 & 0,274 & 0,275 & 0,272 & 0,271 & 0,271 & 0,275 & 0,272 & 0,269 & 0,273 & 0,267 & ,265 \\
\hline & 44 & 420 & 0,435 & 0,443 & 0,436 & 0,420 & 0,426 & 0,429 & 0,425 &, 412 & 0,398 & 0,391 & 0,418 & 0,406 & 0,376 & 372 \\
\hline iás & 112 & 0,406 & 0,416 & 0,418 & 0,407 & 0,400 & 0,392 & 0,390 & 0,389 & 0,390 & 0,389 & 0,392 & 0,404 & 0,396 & 359 & 0,362 \\
\hline arant & 554 & 0,528 & 0,616 & 0,579 & 0,555 & 0,529 & 0,493 & 0,557 & 0,545 & 0,557 & 0,563 & 0,602 & 0,563 & 0,510 &, 596 & 0,558 \\
\hline $\operatorname{tac} c$ & 459 & 0,460 & 0,458 & 0,462 & 0,467 & 0,442 & 0,445 & 0,448 & 0,439 & 0,448 & 0,451 & 0,440 & 0,428 & 0,430 & 0,388 & 0,398 \\
\hline Mato Grosso do & 60 & 0,443 & 0,434 & 0,441 & 0,444 & 0,420 & 0,428 & 0,434 & 0,424 & 0,427 & 0,421 & 0,432 & 0,416 & 0,417 & 94 & 392 \\
\hline & & 3 & & & & 9 & & 8 & 5 & 1 & 9 & 0 & 9 & 19 & & 429 \\
\hline & & 7 & 0 & 1 & 0 & 2 & & 9 & 69 & 44 & 35 & 33 & 8 & 15 & 8 & 16 \\
\hline diva & 40 & 0,603 & 0,602 & 0,590 & 0,617 & 0,564 & 0,578 & 0,563 & 0,573 & 53 & 0,560 & 0,550 & 0,546 & 566 & 46 & 540 \\
\hline ana & 186 & 0,455 & 0,439 & 0,454 & 0,443 & 0,441 & 0,436 & 0,436 & 0,428 & 0,437 & 0,428 & 0,419 & 0,425 & 0,415 & 0,397 & 387 \\
\hline Pernan & 60 & 0,573 & 0,568 & 0,596 & 0,563 & 0,612 & 0,610 & 0,673 & 0,598 & 0,606 & 0,603 & 0,594 & 0,583 & 0,549 & 0,542 & 0,513 \\
\hline uí & 25 & 0,652 & 0,622 & 0,595 & 0,623 & 0,578 & 0,640 & 0,596 & 0,613 & 0,604 & 0,552 & 0,565 & 0,588 & 562 & 19 & 537 \\
\hline & 47 & 0,351 & 0,333 & 0,356 & 0,332 & 0,348 & 47 & 0,350 & 0,351 & 0,354 & 0,359 & 0,359 & 0,351 & 40 & 39 & 336 \\
\hline & 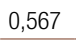 & 0,545 & 0,56 & 0,584 & 0,55 & 0,587 & 0,552 & 5 & 0,531 & 1 & 524 & 4 & 6 & & & 0,492 \\
\hline Gra & 00 & 0 & 0 , & 0,4 & 0,40 & 0,409 & 0,39 & 98 & 2 & 38 & 38 & 34 & 90 & 36 & 6 & 363 \\
\hline ndônia & 454 & 0,467 & 0,486 & 0,454 & 0,444 & 0,443 & 0,460 & 0,455 & 0,568 & 0,569 & 0,539 & 0,533 & 0,530 & 0,530 &, 484 & 0,496 \\
\hline Roraima & 365 & 0,391 & 0,434 & 0,413 & 0,470 & 0,411 & 0,415 & 0,494 & 0,490 & 0,599 & 0,503 & 0,512 & 0,424 & 0,470 & 489 & 441 \\
\hline Santa Catarina & 43 & 0,440 & 0,430 & 0,442 & 0,437 & 0,406 & 16 & 0,418 & 91 & 17 & 13 & 0,402 & 402 & 993 & 73 & 369 \\
\hline 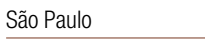 & 347 & 6 & 0,346 & 0,342 & 0,340 & 36 & 0,347 & 50 & c & 4 & 40 & 33 & 36 & 33 & 3 & 3 \\
\hline . & 33 & 0,448 & 0,476 & 0,459 & 0,430 & 0,430 & 0,427 & 0,431 & 0,436 & 106 & 433 & 15 & 426 & 425 & 413 & 7 \\
\hline cantins &, 575 & 0,678 & 0,590 & 0,581 & 0,535 & 0,552 & 0,557 & 0,570 & 0,557 & 0,575 & 0,544 & 0,536 & 0,535 & 0,526 & 0,542 & 0,50 \\
\hline
\end{tabular}

Fuente: Elaboración propia.

Nota: Todos los valores son significativos al $1 \%$.

Cuadro A1.3

Estadísticas descriptivas de la muestra

\begin{tabular}{|c|c|c|c|c|}
\hline Variable & Media & $\begin{array}{l}\text { Desviación } \\
\text { estándar }\end{array}$ & Mínimo & Máximo \\
\hline Entropía & 0,47 & 0,085 & 0,33 & 0,74 \\
\hline PIB real per cápita (en miles de millones de reales)a & 8,70 & 5,746 & 1,41 & 32,44 \\
\hline Gasto real en salud per cápita (en millones de reales) & 390,50 & 901,000 & 5,20 & 7758,00 \\
\hline Gasto real en educación per cápita (en millones de reales) & 292,20 & 578,800 & 1,50 & 5479,00 \\
\hline Años de estudio de los hombres & 7,33 & 1,272 & 4,20 & 10,48 \\
\hline Años de estudio de las mujeres & 8,46 & 1,224 & 4,91 & 11,31 \\
\hline Grado de informalidad & 0,21 & 0,044 & 0,11 & 0,44 \\
\hline
\end{tabular}

Fuente: Elaboración propia.

Nota: PIB: Producto interno bruto.

a Valores en logaritmo natural. 